Revue musicale OICRM

\title{
Capture synchrone de la production sonore et de la gestuelle du pianiste. Vers une interprétation
}

\section{Alain Villard, Floren Colloud et Jean-Christophe Valière}

Volume 6, numéro 1, 2019

URI : https://id.erudit.org/iderudit/1062433ar

DOI : https://doi.org/10.7202/1062433ar

Aller au sommaire du numéro

\section{Éditeur(s)}

Observatoire interdisciplinaire de création et recherche en musique (OICRM)

\section{ISSN}

2368-7061 (numérique)

Découvrir la revue

Citer cet article

Villard, A., Colloud, F. \& Valière, J.-C. (2019). Capture synchrone de la production sonore et de la gestuelle du pianiste. Vers une interprétation. Revue musicale OICRM, 6(1), 160-170. https://doi.org/10.7202/1062433ar
Résumé de l'article

Une expérience de mise en évidence du rapport entre gestuelle du pianiste et production sonore induite a été réalisée dans un auditorium dédié à la musique orchestrale. Quatre pianistes de parcours et de styles différents ont exécuté quatre pièces extraites des Kinderszenen, op. 15 (Scènes d'enfants) de Schumann, qui présentent à la fois une homogénéité musicale, mais aussi une grande variabilité d'atmosphères sonores. Les mesures acoustiques mises en place visaient à capter le signal sonore à l'intérieur et à l'extérieur du piano dans l'objectif d'extraire des descripteurs acoustiques par traitement du signal et à réaliser des tests d'écoute du type de ceux effectués en psychoacoustique. Simultanément et de manière synchronisée, la gestuelle des pianistes a été enregistrée par un système de capture de mouvement (MOCAP) composé de caméras haute fréquence ultrasensibles. Les pianistes étaient instrumentés par des marqueurs réfléchissants placés sur une combinaison ou directement à même la peau dans le cas des mains et des doigts. Les premiers résultats des tests psychoacoustiques montrent que l'occurrence des timbres attribués aux extraits sonores est conforme à l'occurrence publiée dans la littérature. 


\title{
Capture synchrone de la production sonore et de la gestuelle du pianiste. Vers une interprétation
}

\author{
Alain Villard, Floren Colloud \\ et Jean-Christophe Valière
}

\begin{abstract}
Résumé
Une expérience de mise en évidence du rapport entre gestuelle du pianiste et production sonore induite a été réalisée dans un auditorium dédié à la musique orchestrale. Quatre pianistes de parcours et de styles différents ont exécuté quatre pièces extraites des Kinderszenen, op. 15 (Scènes d'enfants) de Schumann, qui présentent à la fois une homogénéité musicale, mais aussi une grande variabilité d'atmosphères sonores. Les mesures acoustiques mises en place visaient à capter le signal sonore à l'intérieur et à l'extérieur du piano dans l'objectif d'extraire des descripteurs acoustiques par traitement du signal et à réaliser des tests d'écoute $\mathrm{du}$ type de ceux effectués en psychoacoustique. Simultanément et de manière synchronisée, la gestuelle des pianistes a été enregistrée par un système de capture de mouvement (MOCAP) composé de caméras haute fréquence ultrasensibles. Les pianistes étaient instrumentés par des marqueurs réfléchissants placés sur une combinaison ou directement à même la peau dans le cas des mains et des doigts. Les premiers résultats des tests psychoacoustiques montrent quel'occurrence des timbres attribués aux extraits sonores est conforme à l'occurrence publiée dans la littérature.
\end{abstract}

Mots clés : capture du mouvement ; perception sonore ; piano ; timbre ; traitement du signal acoustique.

\begin{abstract}
An experiment aiming to highlight the relationship between the pianist's gesture and sound production was performed in an auditorium dedicated to orchestral music. Four pianists from different backgrounds and styles performed four pieces of Kinderszenen, op. 15 (Scenes from Childhood) by Schumann, which show both musical homogeneity but also a great variability of sound atmosphere. The capture of the sound signal inside and outside the piano was performed in order to extract identifiers by signal processing and to perform listening tests of those carried out in the field of psychoacoustics. Simultaneously, the pianist's gestures were recorded by a motioncapture system composed of ultrasensitive high frequency cameras. The pianists were instrumented with reflective markers placed on a no reflective suit or directly on the skin in the case of hands and fingers. The first of psychoacoustic's test results show that the occurrence of timbre attributes is coherent of those found in the literature.
\end{abstract}

Keywords: acoustic signal processing; motion-capture; piano; sound perception; timbre. 


\section{OBJECTIFS DE L'EXPÉRIENCE ${ }^{1}$}

Il est acquis de façon empirique par les musiciens que le son résultant de l'exécution d'une œuvre musicale est dépendant du geste, comme par exemple l'attaque des cordes au plectre ou à l'archet pour les instruments à cordes, la pose des doigts, le frappé de peau pour les instruments à percussion ou encore les fluctuations du souffle pour les instruments à vent. Si cet état de fait semble presque évident pour les instruments où l'interaction entre le geste et le système résonant est directe, cela semble plus spéculatif pour le piano pour lequel le son est obtenu au moyen d'un système mécanique très sophistiqué dont la conception s'est affinée au cours du temps, depuis les premiers pianofortes $\mathrm{du} \mathrm{XVIII}^{\mathrm{e}}$ siècle jusqu'au piano moderne, dans l'objectif, entre autres, d'obtenir une homogénéité et une régularité dans le son produit, et ce quel que soit le pianiste. Mais force est de constater, non seulement pour les pianistes eux-mêmes pour lesquels c'est une chose évidente, mais aussi pour les auditeurs, que chaque musicien possède une sonorité bien particulière et souvent très distinctive. Cependant, cette intuition ne peut, en général, pas se vérifier, les conditions d'exécution n'étant jamais les mêmes et les pianos étant différents, tout comme les salles ou les studios.

Initialement et principalement exploratoire, cette expérience vise à mettre en relation directe la capture des gestes et les prises de son indépendamment des aspects mécaniques intermédiaires. Pour ce faire, celle-ci a été réalisée dans un auditorium dédié à la musique orchestrale avec quatre pianistes jouant les mêmes œuvres sur le même piano dans exactement les mêmes conditions acoustiques (Varambon 2018). Les musiciens ont joué à tour de rôle plusieurs courtes œuvres, leur gestuelle étant captée par un système de capture de mouvement et les sons résultants enregistrés à l'aide de microphones de qualité professionnelle et deux têtes artificielles. Si l'objectif de cette étude est simple dans sa formulation, en réalité il y a tout un processus sous-jacent intégrant les choix esthétiques des pianistes ou les stratégies cognitives qui ne peuvent pas être directement accessibles par cette expérience. Comme toute recherche relative à un phénomène humain plurifactoriel complexe, il est nécessaire de réaliser des études partielles visant à observer une direction particulière en fixant autant que possible les autres dimensions. L'innovation de l'expérience présentée dans ce texte réside donc essentiellement dans la synchronisation entre geste et production sonore d'œuvres courtes du répertoire classique jouées par quatre pianistes professionnels avec un piano de concert dans un auditorium dédié à la musique orchestrale.

\footnotetext{
1 Protocole d'éthique : l'ensemble des participants à l'expérience, musiciens comme chercheurs, ont signé l'autorisation de diffusion de leur image par vidéo et photo. L'expérience était conduite en respect des règles de sécurité de l'auditorium sous la responsabilité de la mairie de Poitiers ; un officier de sécurité était présent à proximité et veillait au respect de nos engagements et des horaires. Les appareils de mesures utilisés ainsi que le dispositif de support font l'objet d'une déclaration auprès des services d'hygiène et de sécurité $\mathrm{du}$ laboratoire sous l'égide du CNRS. Les personnels entrants du laboratoire (stagiaires, doctorants) sont soumis à un questionnaire signé par les responsables sur les risques. Les systèmes de capture des signaux (mouvement et son) ne présentent aucun caractère invasif ni dangerosité pour l'homme, ni radiative, ni chimique, ni sonore. Le niveau sonore auquel ont été soumis les sujets de l'expérience psychoacoustique est très inférieur à la norme européenne de $85 \mathrm{~dB}(\mathrm{~A})$ en niveau équivalent ramené sur huit heures.
} 


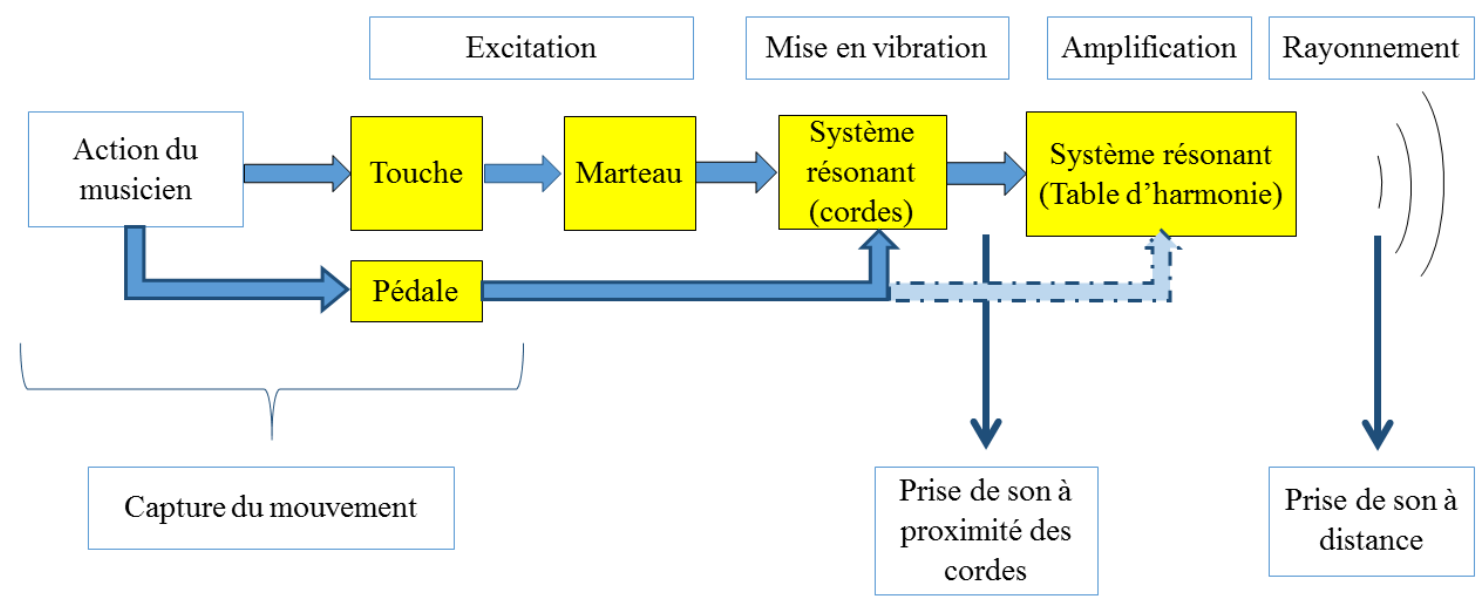

Figure 1: Description de la chaîne opératoire de la production pianistique et de la capture des données physiques.

La figure 1 décrit la chaîne opératoire de la production du son du piano et de la capture des données physiques au cours de l'expérience. Le pianiste par l'ensemble de sa gestuelle produit une accélération sur la touche de piano, avec une certaine liberté dans la position de contact, qui se transmet via un mécanisme complexe au marteau qui frappe la corde et retourne à sa position initiale. L'action de retrait de la touche amène une extinction rapide de la note, sauf si la pédale est actionnée par le pied du musicien, auquel cas les tampons étouffoirs restent relevés. Par cette action, l'ensemble des cordes du piano sont libres de vibrer, engendrant une excitation de la table d'harmonie plus étendue (flèche en pointillé) que lorsque que les touches jouées restent seules appuyées.

Une autre pédale (sourdine ou una corda) permet quant à elle d'accélérer la décroissance de la note, l'action du marteau étant décalée latéralement pour ne frapper qu'une ou deux cordes au lieu de deux ou trois (sur un piano à queue). Car selon la hauteur de la note, le marteau impacte une corde (basses fréquences), deux cordes (fréquences moyennes) ou trois cordes (fréquences aigües) pour compenser à la fois l'intensité du son mais aussi la différence d'inertie qui varient toutes les deux en fonction du diamètre de la corde. L'objectif des facteurs de piano a été de rendre la tessiture de l'instrument la plus homogène possible.

Les vibrations des cordes se transmettent à la table d'harmonie via le chevalet qui joue le rôle classique d'amplificateur comme pour la plupart des instruments à cordes. Elle est la source essentielle du rayonnement qui se poursuit à travers la géométrie du piano.

Si le processus mécanique de production du son, de la touche à la table d'harmonie, a bien été étudié dans la littérature, le son produit ne peut se résumer simplement par la simple superposition des états successifs de l'action du pianiste sur les touches. En effet, plusieurs mécanismes dans la production du son du piano résultent de couplages et d'interactions complexes (marteau/cordes ou cordes/table d'harmonie) qui associés à l'inharmonicité des vibrations des cordes produisent un comportement non linéaire pour lequel le principe de superposition est délicat à utiliser (Chaigne et Kergomard 2013).

En résumé, la variabilité des attaques possibles, la liaison entre les notes, l'usage de la pédale sur des temps plus ou moins longs et enfin la relation entre l'écoute du 
pianiste et sa propre action dans un environnement donné entraînent une sonorité spécifique.

\section{TRAVAUX ANTÉRIEURS ET POSITION DE LA PRÉSENTE ÉTUDE}

Par le statut qu'il occupe dans la musique occidentale, le piano a fait l'objet d'une attention soutenue depuis longtemps tant sur les aspects mécaniques (vibratoire et acoustique) que sur les aspects concernant la perception. Il n'est pas possible de rendre compte dans cette note de l'ensemble de la bibliographie concernant cet instrument, le lecteur se référera avantageusement aux thèses de Chabassier (2012) pour les aspects mécaniques et de Bernays (2013) pour les aspects perceptifs, ainsi que les ouvrages classiques de Askenfelt et Janson (1990) et Chaigne et Kergomard (2013) pour une description physique plus globale.

De très nombreuses études ont été effectuées sur les aspects cognitifs et biomécaniques depuis Ortmann (1929). La thèse de Bernays (2013) et les articles de synthèse de Furuya et Altenmüller (2013) ou Goebl (2018) proposent à ce propos une bibliographie très complète. La bibliographie met en évidence que, à ce jour, seules quelques études impliquent une capture de la gestuelle du pianiste par un système de capture du mouvement. Le coût de cette technologie conjugué aux contraintes qu'elle impose en limite l'accès, malgré la richesse des données obtenues. Les occlusions des marqueurs placés sur les doigts lors du chevauchement des mains ou le passage du pouce sous l'index sont la contrainte la plus difficile à résoudre. En conséquence, l'utilisation d'un piano de concert est encore considérée de nos jours comme un défi (Goeb1 2018). Les conditions expérimentales mises en œuvre dans ces études sont très éloignées d'une condition de concert. Elles sont systématiquement réalisées avec des pianos électriques, lors de mouvements répétitifs n'impliquant que deux doigts (se reporter à Furuya et Altenmüller 2013) ou de courtes mélodies spécialement composées ne requérant qu'une seule main (Della Bella et Palmer 2011). De plus, les marqueurs ne sont placés que sur les membres supérieurs et les doigts, interdisant toute mise en relation entre la gestuelle complète du pianiste et la production du son.

Faire une prise de données complète de l'ensemble de la chaîne opératoire nous semblait trop fastidieux, nous avons donc choisi de privilégier la relation directe entre gestuelle et sonorité sans prise de données sur la mécanique du piano, qui a déjà été bien étudiée. Il a donc été nécessaire, le plus possible, de neutraliser les variations des aspects mécaniques (vibratoire et acoustique) dans la chaîne opératoire. Pour cela, le piano, la salle et les dispositifs de mesures n'ont pas été modifiés au cours de l'expérience. De plus, un répertoire précis a été défini.

\section{CONDITIONS ARTISTIQUES DE L’EXPÉRIENCE}

Il nous a semblé important de centrer notre expérience autour d'une œuvre musicale d'une qualité artistique indiscutable. Nous ne pouvons dissocier notre recherche d'une exigence et d'un contenu artistique réel. Pour cela, nous avons choisi quatre des Scènes d'enfants, op. 15 de Robert Schumann (1839) : "Von fremden Ländern und Menschen » ("Gens et pays étrangers »), "Kuriose Geschichte » ( Curieuse histoire »), "Hasche-Mann » ("Colin-maillard ») et "Wichtige Begebenheit » 
("Un événement important »). Ces quatre pièces, au-delà d'un contenu artistique évident, présentaient l'intérêt d'être brèves, d'offrir des modes de jeux très caractéristiques sur lesquels nous pouvions envisager d'obtenir des constatations suffisamment claires et distinctes sur les gestes employés par les pianistes et leurs rendus sonores, afin d'être différenciés par tests auditifs, traitement des signaux et analyse de la gestuelle.

D'une esthétique commune, ces pièces ne prétendent cependant pas couvrir l'ensemble des modes de jeux pianistiques, ce qui serait illusoire, mais elles permettent plusieurs types de jeux résumés comme suit au moyen du vocabulaire usuel des pianistes :

- "Von fremden Ländern und Menschen » : legato très chantant avec un accompagnement fondu et une polyphonie dense avec des plans sonores très différenciés et des nuances s'exprimant en douceur ;

- "Kuriose Geschichte »: grande vivacité rythmique amenant à réaliser des gestes de sections très courtes, détachés et alternance d'unissons et de notes très liées entre elles ;

- "Hasche-Mann» : staccato rapide de la main droite, d'une grande vélocité, avec des déplacements de la main gauche qui entraînent des attaques variées et très rapides, produisant ainsi un discours perlé avec des nuances contrastées ;

- "Wichtige Begebenheit ": technique d'accords et d'octaves, induisant la verticalité des gestes pianistiques, production d'une puissance sonore caractéristique d'une déclamation. En style pianistique, le son peut être qualifié de bronzé et compact.

Au-delà de la diversité intrinsèque de l'œuvre de Schumann, les quatre pianistes réunis pour cette expérience sont à la fois concertistes et pédagogues et possèdent des styles de jeux très différenciés issus bien entendu de leur personnalité propre, mais aussi de leur parcours éducatif et professionnel. En particulier, il y a un pianiste américain (Alan Kenneth) ayant travaillé aussi avec un pianiste russe, une pianiste française (Catherine Schneider) ayant travaillé aux États-Unis, un pianiste français (Pierre-Yves Jalicon) ayant été formé par un pédagogue hongrois, et enfin un pianiste français (Alain Villard, également coauteur et coresponsable du projet) ayant travaillé au Canada. De surcroît, les pianistes présentent aussi des caractéristiques anthropomorphiques différentes (taille, dimension des mains, masse, etc.).

Si une grande importance a été portée à cette première étape du choix des œuvres et des pianistes, c'est pour que la caractérisation scientifique des timbres en fonction de la gestuelle s'appuie sur la culture et les habitudes de pianistes professionnels en plein exercice. Notons que l'analyse des variabilités en termes de timbre perçu et de signature spectrale portera autant sur les différences produites par un pianiste au travers des quatre pièces (analyse intra-pianiste) que sur celles réalisées par les quatre pianistes sur le même extrait (analyse inter-pianiste). À partir de ces préalables les moyens techniques sont mis en œuvre dans l'objectif de servir cette compréhension.

Pour l'expérience, nous avons aussi pu bénéficier d'un piano de marque Steinway and Sons, modèle D (concert) prêté par le Théâtre Auditorium de Poitiers (TAP). Vieux de dix ans exactement, il a été accordé pour cette occasion par son accordeur habituel. Nous l'avons placé sur la scène dans la configuration " récital ", c'est-à-dire au centre de l'espace scénique, de façon ainsi à pouvoir placer la structure métallique supportant les 15 caméras composant le système de capture de mouvement. 


\section{Mesures ACOUstiQues}

Les mesures acoustiques ont pour objectif d'obtenir des signaux de pression que l'on peut analyser par des méthodes acoustiques, des signaux binauraux exploitables pour des tests auditifs et des signaux de prise de son de type " audio ", très peu directionnels, pour s'affranchir légèrement du retour de la salle et obtenir des signaux exploitables pour une diffusion grand public.

Deux têtes artificielles Head-Acoustics ${ }^{\circledR}$ sont utilisées, l'une positionnée à côté du pianiste pour reproduire l'audition de celui-ci (type analogique HSU), figure 3, et l'autre à proximité du couple de microphones reproduisant la perception d'une personne à proximité du piano (type numérique HMS), montrée sur la figure 2 (droite). Les têtes artificielles permettent, par intégration et traitement des deux signaux binauraux, de remonter à un signal de pression acoustique et de pouvoir réaliser des traitements des signaux adaptés. Mais avant tout, leurs signaux reproduisant une conformité d'audition binaurale seront utilisés pour des tests d'écoute à l'aveugle et en laboratoire à la manière de ceux effectués en psychoacoustique ${ }^{2}$.
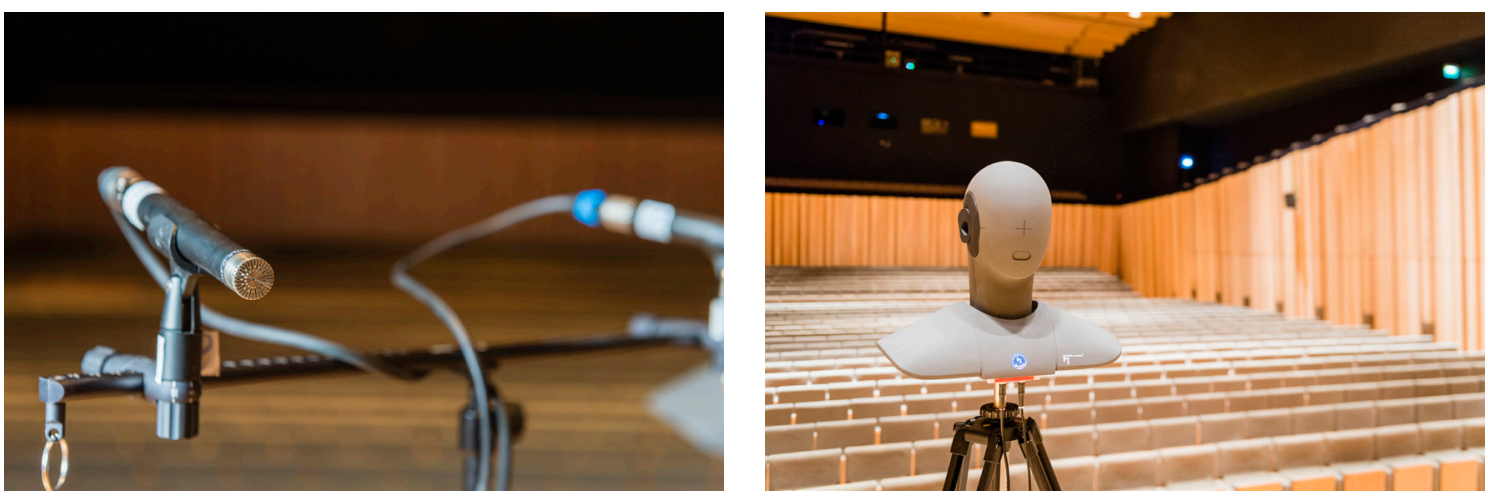

Figure 2: Couple microphonique à gauche, tête artificielle Head-Acoustics à droite. Photos : Cyril FRESILLON / Pprime / CNRS Photothèque.

Les prises de son de type audio sont réalisées au moyen de couples de microphones DPA 4006 TLX, le premier à proximité de l'impact des marteaux pour pouvoir estimer précisément les impacts de notes (meilleure détection des transitoires ; voir figure 3) et l'autre à une distance de $2 \mathrm{~m}$ du piano pour capter le rayonnement du piano dans sa globalité comme le montrent les figures 2 (gauche) et 3.

Il est clair que le son obtenu dépend de la salle, notamment pour les têtes artificielles, mais cela nous a semblé préférable au fait de prévoir l'expérience dans un lieu trop insonorisé par crainte que les pianistes compensent le manque de réverbération par un jeu plus fourni ou plus résonnant comme par exemple un usage trop long de la pédale forte. En parallèle à ces essais, les principales caractéristiques d'auditorium ${ }^{3}$

\footnotetext{
2 La psychoacoustique est une branche de l'acoustique qui vise à quantifier les effets perçus par les auditeurs de stimuli sonores en utilisant les méthodes de la psychologie expérimentale.

3 L'architecte du Théâtre Auditorium de Poitiers (TAP) est le Portugais João Luís Carrilho da Graça et le bureau d'étude acoustique est le Commins Acoustic Workshop.
} 
ont été mesurées. Les résultats donnent un temps de réverbération de 1,8 s calculé en moyenne sur les bandes [250 Hz-2000 Hz], conforme pour un auditorium dédié à la musique orchestrale de cette taille (1000 personnes), et une grande homogénéité des temps de réverbération par bandes d'octaves de $125 \mathrm{~Hz}$ à $4000 \mathrm{~Hz}$. La proximité des capteurs acoustiques permet d'avoir prioritairement le son direct plutôt que le son réverbéré.

Les signaux du jeu de données obtenus par couple microphonique devront être analysés dans l'objectif de trouver des descripteurs acoustiques (temporels ou fréquentiels) qui caractérisent la sonorité distinctive de chaque pianiste, ces signatures pouvant être aussibien globales (spectre moyen) quelocalisées dans le temps et obtenues par des analyses temps/fréquence. L'identification par des moyens de traitement du signal comme décrit ci-dessus ne permettra peut-être pas d'identifier complétement de façon objective les différences inter-pianistes tant le défi est élevé, en particulier l'hypothèse de variations intra-pianistes au cours de l'exécution d'un morceau n'est pas à écarter, mais devrait permettre en revanche d'identifier des différences perçues par les auditeurs sur des extraits dont les timbres sont particulièrement différents.

Les signaux binauraux captés par les têtes artificielles permettront a posteriori d'effectuer des tests auditifs avec un ensemble de participants volontaires qui, à l'aveugle, devront déterminer, dans un premier temps, s'ils différencient correctement chaque pianiste, et dans un second temps, si des caractères sonores particuliers se dégagent pour chacun des pianistes ou extraits choisis.

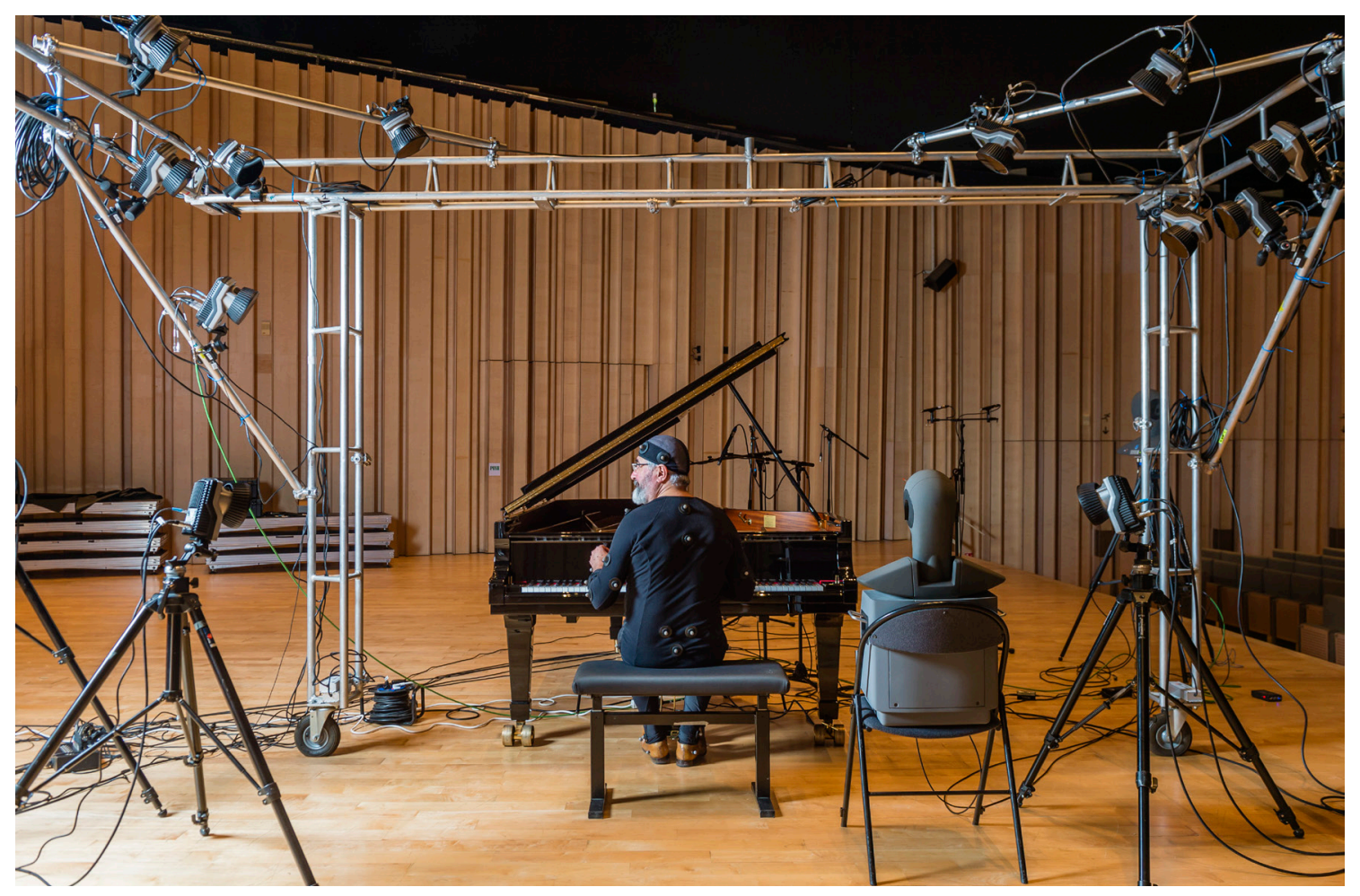

Figure 3 : Vue d'ensemble du dispositif expérimental avec la structure métallique supportant le système de capture de mouvement composé de 15 caméras, les têtes artificielles et les couples de microphones. Photo: Cyril FRESILLON / Pprime / cNRs Photothèque. 


\section{CAPture DU MOUVEMENT}

Les analyses timbrales obtenues, soit par traitement du signal, soit par les tests perceptifs devront être ensuite confrontées aux mouvements des pianistes. Il s'agit donc de recueillir la gestuelle des pianistes comprenant la caractérisation fine de l'attaque de la touche en relation avec les variations temporelles ou fréquentielles des sons produits par les pianistes afin d'établir de manière scientifique les relations entre ces différentes variables.

La situation (position et orientation) de l'ensemble des segments ${ }^{4}$ du pianiste, y incluant les doigts, a été enregistrée par un système de capture du mouvement composé de 15 caméras haute fréquence (Oqus 7+, Qualisys, Suède) captant l'évolution spatiale de marqueurs passifs (sphériques, $\varnothing 12 \mathrm{~mm}$ ) positionnés sur un vêtement évitant les réflexions parasites et à même la peau pour les mains et les doigts (hémisphériques, $\varnothing 3 \mathrm{~mm}$ ) comme le montrent les figures 3 et 4 . Des essais préalables ont été réalisés afin de s'assurer que le placement de ces marqueurs n'altérait pas les possibilités de mouvement des pianistes, notamment au niveau des doigts. Des réflecteurs étaient aussi positionnés sur quelques touches du piano pour pouvoir recaler le corps du pianiste à l'instrument et caractériser plus finement les interactions entre le doigt et la touche dans la production du son. Le nombre de marqueurs placés sur le pianiste est de 90 dont 48 pour les mains (24 par main).

Le système de capture du mouvement est synchronisé au système acoustique et les fréquences d'échantillonnage sont dans un rapport multiple pour faciliter le repérage des évènements temporels, c'est-à-dire de $48 \mathrm{kHz}$ pour l'acoustique et $240 \mathrm{~Hz}$ pour la capture du mouvement.
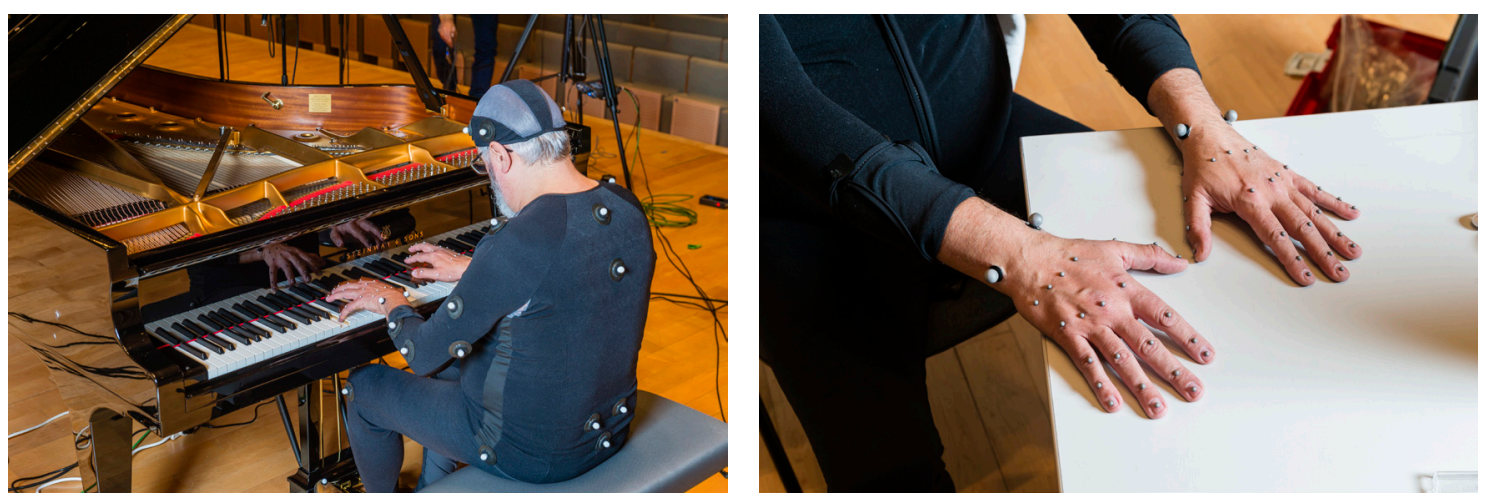

Figure 4 : Position des marqueurs réfléchissant sur le pianiste Alain Villard, à gauche. Détail des mains, à droite. Photos: Cyril FRESILLON / Pprime / cNRS Photothèque.

Le premier travail consiste à reconstruire les trajectoires tridimensionnelles pour les 48 enregistrements ( 4 œuvres courtes $\times 3$ essais $\times 4$ pianistes). Ce travail doit être effectué avec soin du fait des nombreuses configurations et des conditions expérimentales qui entraînent de nombreuses situations d'occlusion.

4 Les segments doivent être entendus au sens d'unités mécaniques élémentaires (par exemple tête, tronc, bassin, cuisse, phalanges des doigts, etc.) dans le cadre d'une modélisation simplifiée du corps humain. 
Notre objectif au cours de cette partie du projet est de caractériser la signature cinématique de chaque pianiste ; 1'hypothèse que nous formulons est qu'au même titre que chaque pianiste peut être caractérisé par le son qu'il produit (signature spectrale), sa gestuelle peut être identifiée par des indicateurs cinématiques telle une signature. La littérature nous fournit des résultats qui confortent cette hypothèse (Dalla Bella et Palmer 2011 ; Furuya et Altenmüller 2013) dans des conditions expérimentales très différentes de celles mises en place pour notre étude. Cette signature sera recherchée au niveau des doigts et du membre supérieur sur la base des coordonnées spatiales des marqueurs et des angles articulaires dans un premier temps. Elle sera étendue au corps du pianiste dans un second temps en se basant sur l'hypothèse que le pianiste joue son instrument avec l'ensemble de son corps. En d'autres termes, il s'agira d'identifier les synergies cinématiques pour chaque pianiste. Les questions auxquelles nous souhaitons répondre portent sur la stabilité de cette signature pour un même pianiste lorsqu'il joue un même morceau plusieurs fois, lorsqu'il joue un morceau différent et si cette signature permet de différencier chaque pianiste.

Notre analyse portera tout d'abord sur les phrases musicales qui ont été isolées lors de l'analyse psychoacoustique. Elle a mis en évidence une signature spectrale identifiant chaque pianiste (voir la section suivante). Pour ce faire, chaque doigt qui joue une note de ces phrases sera identifié. Chaque note jouée sera ensuite caractérisée d'un point de vue spatiotemporel en trois phases à partir de la cinématique du doigt : attaque, enfoncement, relâchement. Nous identifierons la notion d'anticipation entre chaque note. Ces paramètres discrets seront comparés statistiquement par une analyse de variances. Les synergies cinématiques seront, quant à elles, mises en évidence à l'aide d'une analyse en composantes principales (Daffertshofer et al. 2004 ; Dalla Bella et Palmer 2011).

\section{Perspectives}

L'expérience, réalisée les 21 et 22 décembre 2017, a été filmée par une équipe de communication du CNRS (Varambon 2018), les signaux recueillis font actuellement l'objet de post-traitement. L'approche choisie pour l'analyse des données est de commencer par l'évaluation auditive et de finir par l'analyse gestuelle. En effet, il semble pertinent de vérifier dans un premier temps qu'un panel d'auditeurs non-musiciens, musiciens non professionnels ou professionnels soient capable d'identifier des différences entre le jeu des pianistes et aussi de qualifier le timbre des extraits choisis. Le test a déjà été réalisé sur 45 participants dont 10 non-musiciens, 23 musiciens non professionnels, 10 professionnels non-pianistes et 2 pianistes experts, professionnels ou en cycle de professionnalisation. Les tests sont en cours d'analyse, mais déjà les premiers résultats sont confrontés à ceux obtenus dans la littérature (Bernays 2013). Il apparaît que la comparaison directe des pianistes par différenciation par paires est difficile pour tous les sujets même pour les musiciens professionnels (logiquement un peu moins pour les deux pianistes professionnels, mais ils sont statistiquement peu représentatifs). En revanche, le test d'attribution de timbres parmi les 14 identifiés par Bernays et Traube (2011) donne des résultats très encourageants. En effet, les occurrences des adjectifs timbraux obtenues dans nos essais sont fortement corrélées 
avec les occurrences trouvées par les auteurs nommés ci-dessus. Le regroupement en cinq timbres élémentaires renforce encore cette observation. Cela conforte les résultats existants, assez peu nombreux dans la littérature, et valide en même temps la diversité des choix effectués tant des pianistes que des extraits musicaux. Il apparaît de surcroît que les pianistes s'écartent de cette moyenne et possèdent par ce fait des signatures spectrales très différenciées les uns des autres. Les tests montrent aussi que les stratégies timbrales pour la même phrase musicale varient fortement selon les pianistes. Les extraits considérés comme différents par les tests psychoacoustiques sont actuellement étudiés par traitement des signaux acoustiques et par analyse des mouvements.

\section{REMERCIEMENTS}

Les auteurs et coresponsables du projet remercient chaleureusement les trois pianistes Catherine Schneider, Pierre-Yves Jalicon et Alan Kenneth qui ont accepté de se prêter à cette expérience, Frédéric Palpacuer de la société Head-Acoustics qui a fourni une partie du matériel de prise de son binaurale, Danae Le Guennic, étudiante en Master à L'ENSATT de Lyon, ingénieure et musicienne pour la prise de son avec du matériel prêté par Sébastien Noly, ingénieur du son, de la société Sonogramme, Jean-Pierre Manceau pour l'accord du piano, ainsi que Maëva Retailleau, doctorante à 1'Université de Poitiers pour son aide dans la capture des gestuelles. Tout ce projet n'aurait pas pu avoir lieu sans le soutien des institutions, le Théâtre Auditorium Scène Nationale de Poitiers qui a très aimablement accepté de nous recevoir trois jours dans ses murs et qui nous a prêté le piano Steinway Concert Grand, le Conservatoire de région et le CNRS, et en particulier son service audio-visuel de Meudon pour le film et la couverture photo. Ce projet est soutenu par une Action Incitative de l'Institut Pprime en 2018.

\section{BIBLIOGRAPHIE}

Askenfelt, Anders, et Erik Janson (1990), « From Touch to String Vibrations », dans Anders Askenfelt (dir.), Five Lectures on the Acoustics of the Piano, Royal Swedish Academy of Music, https://www.speech.kth.se/music/5 lectures/contents.html, consulté le 24 avril 2019.

Bernays, Michel (2013), « The Expression and Production of Piano Timbre. Gestural Control and Technique, Perception and Verbalisation in the Context of Piano Performance and Practice ", thèse de doctorat, Université de Montréal, https://papyrus.bib.umontreal.ca/xmlui/ handle/1866/10208?locale-attribute=en\&show=full, consulté le 28 mai 2019.

Bernays, Michel, et Caroline Traube (2011), « Verbal Expression of Piano Timbre. Multidimensional Semantic Space of Adjectival Descriptors », Aaron Williamson, Darryl Edwards et Lee Bartel Utrechet (dir.), Proceedings of the International Symposium on Performance Science (ISPS2011), Utrecht, European Association of Conservatoires, p. 299-304, https://www.researchgate.net/ publication/263657775 Verbal expression of piano timbre Multidimensional semantic space of adjectival descriptors, consulté le 28 mai 2019.

Chabassier, Juliette (2012), "Modélisation et simulation numérique d'un piano par modèles physiques ", thèse de doctorat, École polytechnique de Paris, https://pastel.archives-ouvertes.fr/ file/index/docid/690351/filename/These.pdf, consulté le 28 mai 2019. 
Chaigne, Antoine, et Jean Kergomard (2013), Acoustique des instruments de musique, $2^{\text {nde }}$ éd., Paris, Belin. Traduit en anglais en 2016 sous le titre Acoustics of Musical Instruments, Berlin, Springer.

Daffertshofer, Andreas, et al. (2004), " PCA in Studying Coordination and Variability. A Tutorial», Clinical Biomechanics, vol. 19, $\mathrm{n}^{\circ} 4$ (mai), p. 415-428, https://www.sciencedirect.com/science/ article/pii/S0268003304000166?via\%3Dihub, consulté le 28 mai 2019.

Dalla Bella, Simone, et Caroline Palmer (2011), "Rate Effects on Timing, Key Velocity, and Finger Kinematics in Piano Performance ", PLoS ONE, vol. 6, n ${ }^{\circ}$ 6, https://journals.plos.org/plosone/ article?id=10.1371/journal.pone.0020518, consulté le 28 mai 2019.

Furuya, Shinichi, et Eckart Altenmüller (2013), «Flexibility of Movement Organization in Piano Performance ", Frontiers in Human Neuroscience, vol. 7 (juillet), p. 1-10, https://www.frontiersin. org/articles/10.3389/fnhum.2013.00173/full, consulté le 28 mai 2019.

Goebl, Werner (2018), " Movement and Touch in Piano Performance », dans Bertram Müller et Sebastian I. Wolf (dir.), Handbook in Human Motion, Berlin, Springer, p. 1-18, https://iwk. mdw.ac.at/goebl/papers/Goebl2017-SpringerHandbook-PianoMovementTouch-postprint.pdf, consulté le 28 mai 2019.

Ortmann, Otto (1929), Physiological Mechanics of Piano Technique, New York, Dutton.

Schumann, Robert ([1839]2009), Kinderszenen, op. 15, München, G. Henle Verlag.

Varambon, Pascal (2018), Le geste du pianiste, film réalisé pour le cNRs avec partenariat du journal Le Monde, https://lejournal.cnrs.fr/videos/chaque-pianiste-a-t-il-un-son-particulier, consulté le 28 mai 2019. 